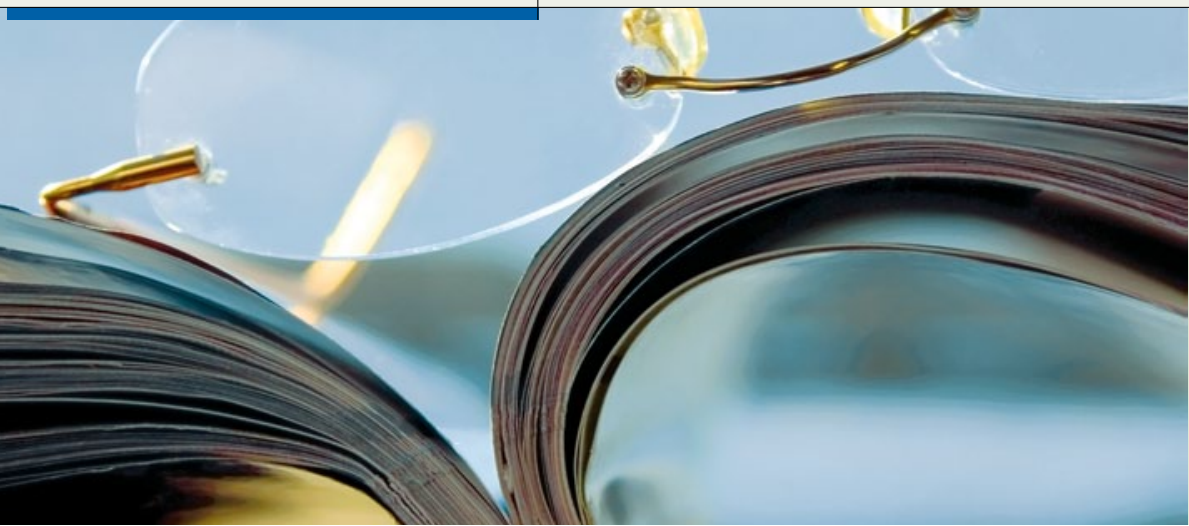

\section{Nanopartikel in Tattoos: Finger weg von Carbon-Schwarz}

Wenn schon tätowieren, dann möglichst nicht in Schwarz: Das lässt sich aus den Ergebnissen einer Studie folgern, in der dänische Forscher den Gehalt von Nanopartikeln in verschiedenen Tattoo-Farben ermittelt haben. Die Farbe Schwarz enthält demnach den höchsten Anteil der Kleinst-Farbteilchen, die als mögliche Kanzerogene gelten.

$T^{2}$ attoos erhalten ihre oft eindrucksvollen Farben durch in die Haut injizierte Pigmente - überwiegend kristalline, wasserunlösliche Partikel, die eine permanente Färbung bewirken. Unklar war bisher, inwieweit die gängigen Tattoo-Farbmittel Nanopartikel, d.h. Teilchen mit einem Durchmesser unter $100 \mathrm{~nm}$, enthalten. Letztere besitzen experimentellen Studien zufolge zumindest toxisches Potenzial, das mit sinkender Partikelgröße offenbar zunimmt. Dänische Autoren haben nun eine Vielzahl verschiedenfarbiger Tattoo-Tinten auf ihren Nanopartikelgehalt untersucht und die jeweiligen Partikelgrößen mittels
Laser- bzw. Röntgenstrahlbeugung und Elektronenmikroskopie bestimmt.

Was überraschte, war der äußerst verbreitete Einsatz von Nanopartikeln in den Tattoo-Tinten, vor allem bei der Farbe Schwarz (Carbon-Schwarz). Diese setzte sich bei fast allen Herstellern nahezu ausschließlich aus Nanopartikeln zusammen. Bei Schwarz fanden sich auch die kleinsten Partikel mit mittleren Teilchendurchmessern von 41-165 nm. Nur bei einer der getesteten Marken maßen die Partikel im Schnitt über $100 \mathrm{~nm}$. Der Grad der Schwärze stieg generell mit abnehmender Partikelgröße.

\section{Phototherapie bei Neugeborenen lässt Melanozyten wuchern}

\begin{abstract}
- An 15 eineiigen und 43 zweieiigen Zwillingspaaren zwischen drei und 30 Jahren untersuchten Forscher, ob ein Zusammenhang zwischen einer Phototherapie in den ersten Lebenstagen und der späteren Anzahl melanozytärer Nävi besteht. Das Besondere an diesen Zwillingspaaren war, dass kurz nach der Geburt nur jeweils ein Geschwisterteil mit blauem kurzwelligem Licht bestrahlt worden war. Die Kinder, die unter einer Phototherapielampe gelegen hatten, wiesen später signifikant mehr melanozytäre Nävi auf der Haut auf als diejenigen, die der Bestrahlung nicht ausgesetzt waren. Ähnliches zeigte sich auch am Auge: Die Blaulichttherapie war mit einer deutlich höheren Prävalenz melanozytärer Veränderungen am Auge assoziiert. Dr. Dagmar Kraus
\end{abstract}

Csoma Z et al. Pediatrics 2011; 128: e856-64
Weiße Pigmente, bestehend aus Titaniumdioxid, waren am größten; hier betrugen die Durchmesser im Mittel zwischen 317 und $738 \mathrm{~nm}$, die kleinsten Größen reichten mit 100-200 nm gerade an den Nanobereich heran. Grüne, blaue, rote und gelbe Pigmente bewegten sich in einem mittleren Bereich zwischen 81 (blau) und 144 bzw. 154 nm (gelb).

Wie die Forscher betonten, trägt die Charakterisierung der in Tattoos enthaltenen Farbpartikel entscheidend dazu bei, deren toxisches Potenzial für den Menschen einzuschätzen. Carbon-Schwarz wurde von der International Agency for Research on Cancer als mögliches Karzinogen eingestuft. Azofarbstoffe, in der vorliegenden Studie in roten und gelben Tinten enthalten, dürfen in Deutschland nicht in Tattoo-Farbmitteln eingesetzt werden.

Fazit: Unter den Tattoo-Tinten enthält die Farbe Carbon-Schwarz den höchsten Anteil an Nanopartikeln und die kleinsten Teilchendurchmesser. Inwieweit sich daraus ein Gefährdungspotenzial für den Menschen ergibt, ist nicht geklärt. Bislang wurden lediglich in experimentellen Studien Entzündungsreaktionen und eine leicht erhöhte Mutationsfrequenz nach Langzeitexposition gegenüber CarbonSchwarz-Partikeln nachgewiesen. Diese Wirkung war indirekt proportional zur Teilchengröße und wurde von den Autoren auf die Bildung reaktiver Sauerstoffderivate zurückgeführt. Ein Bericht der Europäischen Kommission von 2009 fasst den aktuellen Kenntnisstand zusammen: www.nanowerk.com/nanotechnology/ reports/reportpdf/report133.pdf.

Dr. Elke Oberhofer

Hogsberg T et al. Tattoo inks in general usage contain nanoparticles. Br J Dermatol 2011; 165: $1210-18$ 